\title{
Analysis of the clinical value of multi-slice spiral computed tomography (MSCT), magnetic resonance imaging (MRI) and ultrasound (US) in the diagnosis of retroperitoneal tumors
}

\author{
Yuanchun Feng ${ }^{1}$, Wei Zhang ${ }^{1}$, Chenghua Luo ${ }^{2}$ \\ ${ }^{1}$ Department of Radiology, Peking University International Hospital, Beijing, China; ${ }^{2}$ Department of Retroperitoneal Tumor, Peking University \\ International Hospital, Beijing, China \\ Contributions: (I) Conception and design: Y Feng; (II) Administrative support: Y Feng; (III) Provision of study materials or patients: W Zhang; (IV) \\ Collection and assembly of data: C Luo; (V) Data analysis and interpretation: W Zhang, C Luo; (VI) Manuscript writing: All authors; (VII) Final \\ approval of manuscript: All authors. \\ Correspondence to: Yuanchun Feng, MD. Department of Radiology, Peking University International Hospital, No. 1 life Garden Road, Zhongguancun \\ Life Science Park, Changping District, Beijing 103211, China. Email: ycfeng20200413@163.com.
}

\begin{abstract}
Background: To compare the differences in the diagnosis of retroperitoneal tumors among multi-slice spiral computed tomography (MSCT), magnetic resonance imaging (MRI), and ultrasound (US).

Methods: Sixty cases of retroperitoneal tumors admitted in our hospital from January 2016 to January 2019 were collected and related data were analyzed. After admission, patients were examined by MSCT, MRI, and US, and the pathological results of the patients were used as the controls. The differences in the diagnosis of retroperitoneal tumors were compared with the results of MSCT, MRI, and US.

Results: Thirteen cases of benign tumors were diagnosed by MSCT, 47 cases were malignant, and 1 case was false benign, with diagnosis accuracy, sensitivity and specificity of $98.33 \%, 97.92 \%$ and $92.30 \%$, respectively. Thirteen cases of benign tumors were diagnosed by MRI, 47 cases of malignant tumors, and 1 case was false benign, with diagnosis accuracy, sensitivity and specificity of $98.33 \%, 97.92 \%$, and $92.30 \%$, respectively. Fourteen cases of benign tumor were diagnosed by US, 46 cases were malignant, and 2 cases was false benign, with diagnosis accuracy, sensitivity and specificity of 96.67\%, 97.92\%, and 85.71\%, respectively. There were no statistically significant differences in the accuracy, sensitivity, and specificity of MSCT, MRI, and US in the diagnosis of retroperitoneal tumors $(\mathrm{P}>0.05)$.
\end{abstract}

Conclusions: MSCT, MRI, and US tests are highly accurate, sensitive, and specific in the diagnosis of retroperitoneal tumors.

Keywords: Multi-slice spiral computed tomography (MSCT); magnetic resonance imaging (MRI); ultrasound (US); retroperitoneal tumor

Submitted Oct 24, 2020. Accepted for publication Mar 12, 2021.

doi: $10.21037 /$ tcr-20-3141

View this article at: http://dx.doi.org/10.21037/tcr-20-3141

\section{Introduction}

Retroperitoneal tumors are tumors that originate in the retroperitoneal space (including retroperitoneal fat, connective tissue, fascia, blood vessels, muscles, nerves, lymph and embryonic residual tissues). Because the tumor grows deep, it is affected by organs in the abdominal cavity, and the symptoms are hidden, the patient's condition has been relatively severe at the time of consultation (1). The clinical incidence of retroperitoneal tumors is not high, but $85 \%$ of tumors that occur retroperitoneally are malignant $(2,3)$. The retroperitoneal cavity has a complex anatomical structure and a wide range of involvement, which is difficult to detect early, but early detection and effective treatment are of great significance for prognosis and can improve 
survival (4).

Multi-slice spiral computed tomography (MSCT) has been used commercially for more than two decades for the diagnosis and screening of various diseases $(5,6)$. MSCT plain scan combined with enhanced scan images could well show the location, size, extent of the lesion and the adjacent relationship with the surrounding structure (pushing displacement, enveloping and invasion), but also the internal density of the lesion (solid and cystic and calcification, internal division of fatty lesions, solid nodules or masses, cystic foci), and enhanced scanning (irregularly enhanced morphology, internal necrosis) should be observed. Magnetic resonance imaging (MRI) offers superior soft-tissue contrast as compared with computed tomography (CT) (7). The components of the MRI inside the lesion (common heterogeneity of retroperitoneal tumors) had a higher display ability, and could clearly show the fibrous separation in fatty lesions, tumor fat and minor abnormal signal changes combined with other decomposed components, the shape of the edges, and the relationship between them and their surroundings. Ultrasound (US) technology is currently growing exponentially due to its many advantages of improved and real-time high-resolution US imaging that results in successful pain management interventions (8). The US can determine the anatomical location of the tumor, the subtle relationship between the tumor and adjacent structures (slightly affected), and it is especially important to observe adhesion or involvement with large blood vessels or ureteral walls.

In this study, MSCT, MRI, and US are used to investigate the diagnosis of retroperitoneal tumors, providing a reference for the clinical diagnosis of retroperitoneal tumors.

We present the following article in accordance with the STARD reporting checklist (available at http://dx.doi. org/10.21037/tcr-20-3141).

\section{Methods}

\section{Patients}

A retrospective study was performed. Sixty cases of retroperitoneal tumors underwent MSCT, MRI, and US examinations in our hospital from January 2016 to January 2019 were selected as subjects, and the results of MSCT, MRI, and US examinations were compared with corresponding pathological examination results. The study was conducted in accordance with the Declaration of Helsinki (as revised in 2013). The study was approved by the Ethics Committee of Peking University International Hospital and informed consent was taken from all the patients.

The male to female ratio was $0.9: 1$, and the average age was $47 \pm 2.7$ years. Exclusion criteria: (I) patients with other malignant tumors; (II) pregnant women; (III) not suitable for MSCT, MRI, US examination; (IV) patients with severe cardiovascular and cerebrovascular disease; (V) patients with unconsciousness, claustrophobia.

\section{MSCT examination}

Siemens Sensation dual-source CT was used for MSCT examination, the scanning layer thickness was $10 \mathrm{~mm}$, and the layer distance was $10 \mathrm{~mm}$. Reconstructed layer thickness and interval was $1 \mathrm{~mm}$. The scan ranged from the diaphragm to the pubic bone and included the entire abdominal and pelvic region (generally suitable for those with large lesions occupying the entire abdominal and pelvic cavity, and can also include smaller retroperitoneal tumors to determine the presence of intraperitoneal metastases). Scan technical parameters: plain scan, the scanning layer thickness was $5 \mathrm{~mm}$, and the layer distance was $5 \mathrm{~mm}$. Reconstructed layer thickness and interval was $1 \mathrm{~mm}$. Scan parameters: contrast injection of elbow vein $(80 \mathrm{~mL})$, speed of $3.5 \mathrm{~mL} / \mathrm{s}$, dual-phase dynamic enhanced scan. Patients' images were transferred to the PACS system.

\section{MRI examination}

Scanning was performed using a GE 3.0T MRI scanner. The patient was lying flat with head first, and body coils covered the entire lesion area (for smaller lesions in the upper abdomen or pelvic area). Patient should keep breathing smoothly, and fasted for 6-8 hours before the examination. Scan parameters: axial plain scan, enhanced axial, sagittal, and coronal scans (elbow vein injection of gadopentetate glucosamine, dose of $0.2 \mathrm{mg} / \mathrm{kg}$, speed of $2 \mathrm{~mL} / \mathrm{s}$ ). MRI sequences: fast spin echo (FSE) T1-weighted imaging (TIWI), fat-suppressed T1-weighted imaging (T1WI-FS), in-phase and oppose-phase T1WI; T2WI-FS, diffusion-weighted imaging (DWI) and apparent diffusion coefficient (ADC), dynamic enhanced T1WI-FS. Patients' images were transferred to the PACS system.

\section{US examination}

The US examination was performed with a Philips iU 
Table 1 Malignant and benign features of retroperitoneal tumors

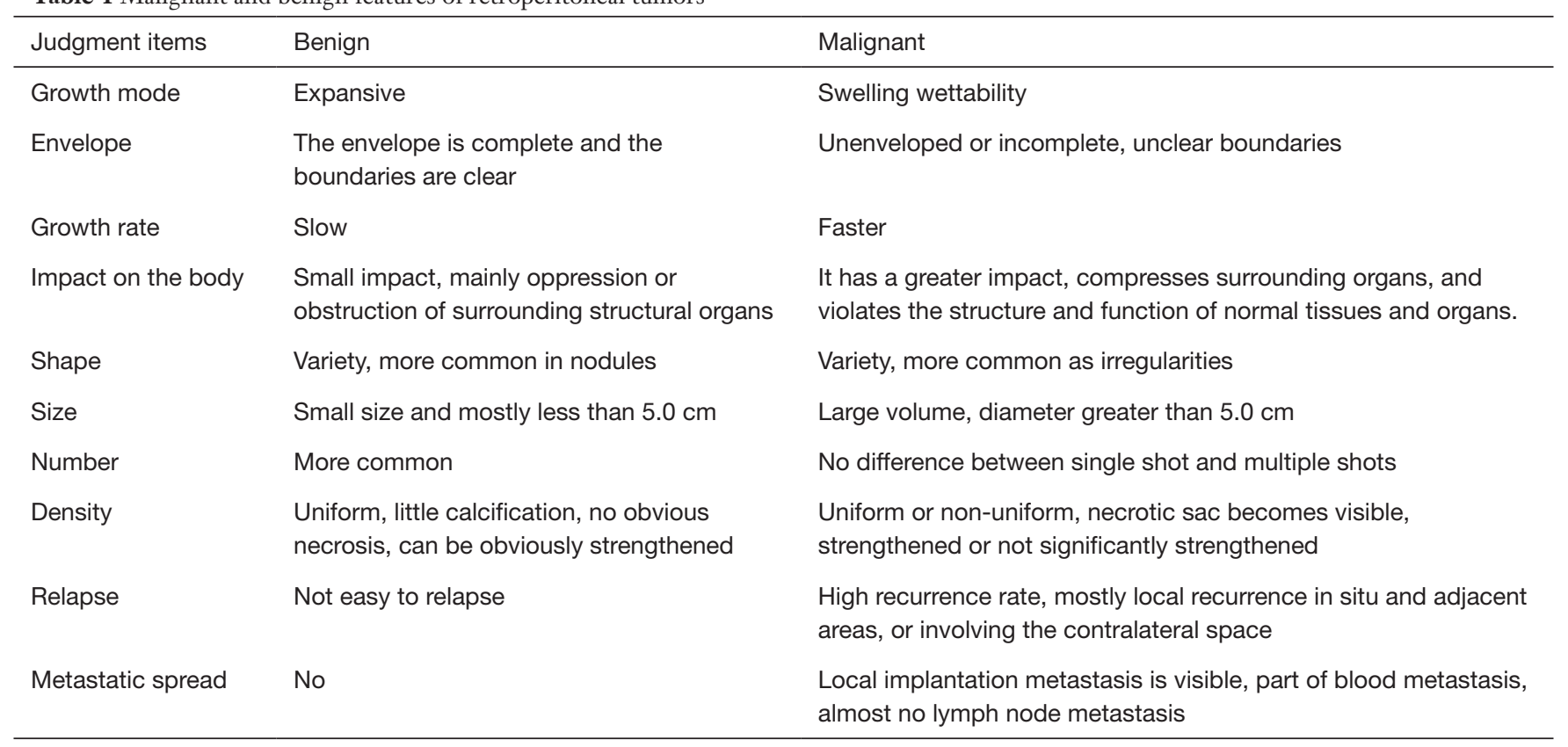

Elite color ultrasound system with a probe frequency of 3.5 MHz. Fasting for 6-8 hours before examination, patients undergo supine, lateral, and prone examinations. The size, shape, boundary, location and internal echo of the mass were observed.

\section{Observation indicators and evaluation criteria}

The clinical data of the selected cases were analyzed, and the accuracy, sensitivity, and specificity of the three examination methods were compared. Among them, accuracy [(case of true malignant and true benign)/all cases], sensitivity (case of true malignant/all cases), and specificity (case of true benign/all cases) were calculated. The malignant and benign diagnostic criteria for retroperitoneal tumors were shown in Table 1. Judgment was based on the growth mode and speed of the tumor, the presence or absence of an envelope, the effect on the body, the shape, number, size, density, whether the tumor has recurred, and whether it has metastasized or spread.

\section{Statistical analysis}

Statistical software SPASS 15.0 was used for statistical analysis of the experimental data. Comparison of MSCT, MRI, and US examination were analyzed by Chi-square test. $\mathrm{P}<0.05$ was considered to be significant.

\section{Results}

\section{Analysis of clinical characteristics of patients}

In this study, 60 patients with retroperitoneal tumors included 19 cases of abdominal discomfort/abdominal pain, 11 cases without clinical symptoms, 11 cases that were found during physical examination or accident, 7 cases of abdominal masses, 5 cases of weight loss, 5 cases of waist pain, 3 cases of leg pain, and 10 cases of other symptoms. Pathological diagnosis results: 12 cases were benign tumor lesions, accounting for $20 \%$; 48 cases were malignant tumors, accounting for $80 \%$, mainly liposarcomas, malignant neurogenic tumors, some fibrous tumors, and musculoskeletal malignancies, malignant gastrointestinal stromal tumor (GIST), embryonic tumor, lymphoma, neuroendocrine tumor (Table 2).

\section{Example results of MSCT, MRI, and US examination}

MSCT, MRI, and US images of a representative patient (female, 15 years old, retroperitoneal cell neuroma) were shown in Figure 1. Axial CT plain scan results: an irregular, slightly low-density mass $(\mathrm{N})$ was seen in the right front of the horizontal spine $(\mathrm{V})$ of the right kidney, 
Table 2 General clinical and pathological information for patients with retroperitoneal tumors

\begin{tabular}{|c|c|c|}
\hline Items & Number & Proportion (\%) \\
\hline \multicolumn{3}{|l|}{ Reason for visiting } \\
\hline \multirow{3}{*}{$\begin{array}{l}\text { Abdominal discomfort/ } \\
\text { abdominal pain }\end{array}$} & 19 & 31.67 \\
\hline & 11 & 18.33 \\
\hline & 7 & 11.67 \\
\hline $\begin{array}{l}\text { Physical examination } \\
\text { (incidentally found) }\end{array}$ & 5 & 8.33 \\
\hline \multirow[t]{2}{*}{ Abdominal mass } & 5 & 8.33 \\
\hline & 3 & 5.00 \\
\hline Weight loss & 10 & 16.67 \\
\hline \multicolumn{3}{|l|}{ Pathology } \\
\hline Liposarcoma & 24 & 40 \\
\hline Neurogenic tumor $^{1}$ & 11 & 18.33 \\
\hline Musculoskeletal tumor ${ }^{2}$ & 9 & 15 \\
\hline Lymphoma & 2 & 3.33 \\
\hline Others $^{3}$ & 14 & 23.33 \\
\hline
\end{tabular}

${ }^{1}$, neurogenic tumors: schwannomas, paraganglioma, neurofibromas, gangliomas, pheochromocytomas, malignant peripheral schwannomas, and low-grade mural schwannoma; ${ }^{2}$, musculoskeletal tumors: leiomyosarcoma, rhabdomyosarcoma, synovial sarcoma, osteosarcoma, chondrosarcoma; ${ }^{3}$, others: fibrous tumors (including solitary fibroids, fibromatosis, etc.), GIST, embryonic tumors, lymphatic cysts, teratomas, neuroendocrine tumors, idiopathic fibrosis.

with uniform internal density and clearer boundaries (thick arrows) (Figure 1A). Axial CT enhanced scan of the venous phase: the density within the lesion was uniform, but lower than the density of the kidney $(\mathrm{K})$ and liver $(\mathrm{L})$, with clear boundaries, and partially enclosing the abdominal aorta (A) and the left renal vein (LV) (Figure 1B). Coronary CT enhanced scan of the venous phase: the lesion was irregularly lobulated, with a slight uneven fibrous enhancement in the interior, with clear boundaries, but close to the abdominal aorta (Figure 1C). MRI axial T1WIFS MASK (flat scan) sequence: an irregular slightly signaled mass $(N)$ was seen in the right front of the horizontal spine of the right kidney, and the tumor border was smooth (thick arrow) (Figure 1D). MRI axial T2WI-FS sequence: the lesion showed a long T2 high signal, the internal signal was more uniform, a separable shadow (thin arrow) was visible on the left side, and the tumor border was smooth
(Figure 1E). MRI axial DWI sequence: the mass showed a high signal $(\mathrm{N})$, the internal signal was basically uniform, and the lesion boundary was clearer (white thick arrows) (Figure $1 F$ ). MRI T2WI-FS coronary coronal sequence: The lesion was mainly located on the right side of the lumbar spine, with a long T2 high signal inside and a clearer boundary (Figure 1G). Arterial phase sequence of MRI axial T1WI enhanced scan: the lesion showed a slightly uneven low signal (compared with the renal signal), surrounding the right renal artery and the right part of the abdominal aorta, and the tumor boundary was clear and smooth. The boundary with the abdominal aorta was clear and sharp. The right kidney was partially compressed (Figure 1H). US image: a solid hypoechoic mass with retroperitoneum and uniform internal echo. Enveloped, with clear boundaries and acceptable morphology. Color Doppler flow imaging (CDFI): the little blood flow shown the abdominal aorta behind the tumor. The lesion squeezed the right kidney, and the demarcation was clear (Figure 11).

\section{Comparison of MSCT, MRI, and US examination}

Thirteen benign, 46 malignant, and 1 false benign cases were diagnosed by MSCT. The diagnostic accuracy, sensitivity, and specificity were $98.33 \%, 97.87 \%$, and $92.86 \%$. Thirteen benign, 46 malignant, and 1 false benign cases were diagnosed by MRI. The diagnostic accuracy, sensitivity, and specificity were $98.33 \%, 97.87 \%$, and $92.86 \%$. Twelve benign, 46 malignant, and 2 false benign cases were diagnosed by US. The diagnostic accuracy, sensitivity, and specificity were $96.67 \%, 95.83 \%$, and $85.71 \%$ (Table 3). Statistical analysis found that there were no significant differences in the accuracy, sensitivity, and specificity of MSCT, MRI, and US in the diagnosis of retroperitoneal tumors $(\mathrm{P}>0.05)$ (Table 4). There were no adverse events in MSCT, MRI, and US examination.

\section{Discussion}

The clinical incidence of retroperitoneal tumors is lower than other tumors, but once found, they are mostly malignant $(9,10)$. Early detection and early treatment are essential to improve the quality of life of these patients. With the wide application of imaging technology and rich clinical diagnosis experience, the combination of MSCT, MRI, and US can effectively compensate the diagnostic accuracy of retroperitoneal tumors and help clinical selection of correct treatment measures. The diagnosis 


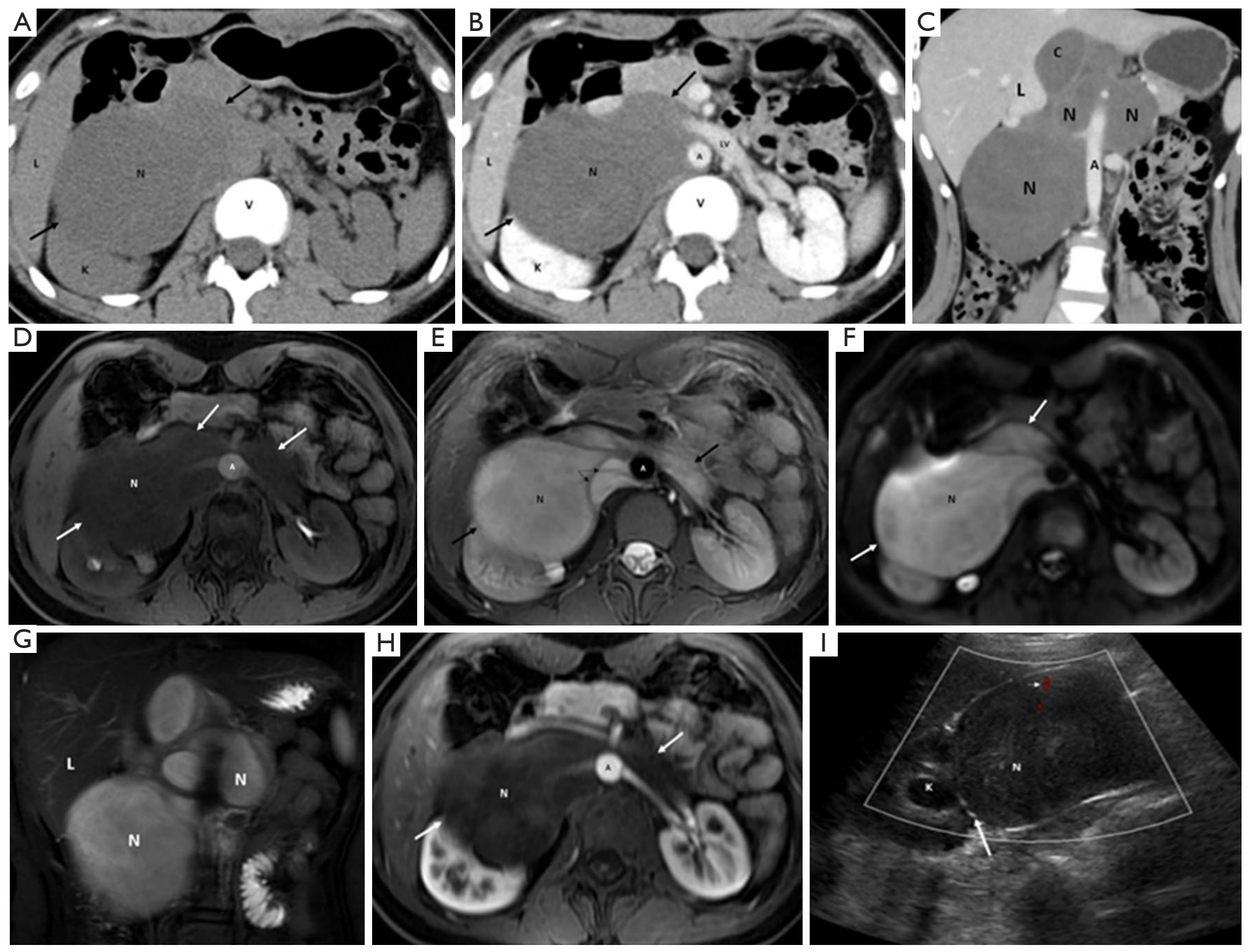

Figure 1 MSCT, MRI, and US images of a representative patient (female, 15 years old, retroperitoneal cell neuroma). (A) Axial CT plain scan. (B) Axial CT enhanced scan of the venous phase. (C) Coronary CT enhanced scan of the venous phase. (D) MRI axial T1WIFS MASK (flat scan) sequence. (E) MRI axial T2WI-FS sequence. (F) MRI axial DWI sequence. (G) MRI T2WI-FS coronary coronal sequence. $(\mathrm{H})$ Arterial phase sequence of MRI axial T1WI enhanced scan. (I) US image. MSCT, multi-slice spiral computed tomography; MRI, magnetic resonance imaging; US, ultrasound; T1WI-FS, fat-suppressed T1-weighted imaging; T2 WI-FS, fat-suppressed T2weighted imaging; DWI, diffusion-weighted imaging; US, ultrasound.

of retroperitoneal tumors is based on the gold standard of pathology. How can imaging be used to distinguish benign from malignant tumors before surgery or puncture? Long-term clinical experience is required. Based on the postoperative or puncture biopsy pathology results of our center, we will make some basic clinical analysis for the preoperative imaging diagnosis of retroperitoneal tumors.

The results of this study showed that MSCT, MRI, and US had high accuracy, sensitivity, and specificity for the diagnosis of retroperitoneal tumors $(\mathrm{P}>0.05)$, and all three can be used as clinically ideal diagnosis methods for retroperitoneal tumors. It can provide certain imaging support for early detection and early treatment. A total of 60 cases were selected in this study. The number of patients with malignant lesions were significantly higher than that of patients with benign lesions, which were confirmed in the previous reports $(11,12)$.

The adaptive surface of MSCT examination is more popular. Through the observation of tumor characteristics, size, growth location, tumor boundary, and the typical 
Table 3 Comparison of pathological diagnosis results by MSCT, MRI, and US

\begin{tabular}{lcccc}
\hline \multirow{2}{*}{ Methods } & Results & \multicolumn{2}{c}{ Pathological diagnosis results } & Benign \\
\cline { 2 - 4 } MRI & Malignant & Malignant & 0 & 12 \\
BSCT & Benign & 47 & 0 & $>0.05$ \\
Malignant & Benign & 1 & 12 & 0.05 \\
US & Malignant & 47 & 0 & 12 \\
\hline
\end{tabular}

MSCT, multi-slice spiral computed tomography; MRI, magnetic resonance imaging; US, ultrasound.

Table 4 Comparison of accuracy, sensitivity, and specificity of MSCT, MRI, and US (\%)

\begin{tabular}{lccc}
\hline Methods & Accuracy & Sensitivity & Specificity \\
\hline MSCT & $98.33 \%[59 / 60]$ & $97.92 \%[47 / 48]$ & $92.31 \%[12 / 13]$ \\
MRI & $98.33 \%[59 / 60]$ & $97.92 \%[47 / 48]$ & $92.31 \%[12 / 13]$ \\
US & $96.67 \%[58 / 60]$ & $95.83 \%[46 / 48]$ & $85.71 \%[12 / 14]$ \\
\hline
\end{tabular}

MSCT, multi-slice spiral computed tomography; MRI, magnetic resonance imaging; US, ultrasound.

characteristics of MSCT images (fat tissues and fluids of different densities), the binding site can indicate the source of tumor tissue. It is very helpful, and MSCT image reconstruction technology can be used to observe the shape of the lesion and the anatomical relationship with nearby organs (13), which is conducive to the evaluation of retroperitoneal tumors. When using MSCT for imaging diagnosis, the first task was to determine the location, size and morphology, density and enhancement method of the tumor, and the edge of the lesion (14), and to further judge the benign and malignant tumors based on these conventional features. Benign tumors are generally small, regular in shape, uniform in mass density, and clear in boundary; malignant tumors are generally larger in size, irregular in shape, unclear in boundary and even invade surrounding structures, and the tumor is prone to necrosis or cystic changes.

Scanning MRI in the transverse axis, coronal, and sagittal positions can determine the anatomical relationship between the tumor and the retroperitoneal space, thereby clarifying the tissue source of the tumor. MRI can determine whether the tumor is benign or malignant based on the morphological changes of the tumor, the abnormal signal of different tissues, and the degree of enhancement after enhancement $(15,16)$. MRI can also initially determine the tumor's invasion of surrounding structures and show an increase in local lymph nodes (17), which is conducive to the clinical staging of retroperitoneal tumors.

Because the internal components of some retroperitoneal tumors are more complicated, on the US sonogram, tumors of different origins can also show similar echoes. In addition, most tumors are large and cannot be viewed in full. The type is accurately determined, but the details (the nuances between different structures) are shown to have other checks that cannot be replaced.

Although all three have high accuracy, diagnostic sensitivity, and specificity, the US can effectively determine the subtle adjacency of the tumor, and it can also determine the internal structure of the tumor more accurately. The cost of the US is low, the inspection is convenient, and can be repeated many times without radiation damage. It is the first choice for many clinical examinations. The disadvantage of US is that the difference in diagnostic results will be affected by the operator's experience, lack of accurate objective indicators, and will also be affected by the tumor location and internal organs, and it is not easy to see the full picture of the tumor. In addition, it can objectively record the current status of the disease, storable stable images, and rich digital information carried by the film, providing essential help for clinical diagnosis and treatment. 
In conclusion, the three diagnostic methods had their own characteristics and advantages, all had high accuracy, sensitivity and specificity. The diagnosis using MR, MSCT, and US can partially determine the benign and malignant tumors of the retroperitoneum, and have significant for clinical diagnosis and treatment of retroperitoneal tumors. Any test method can be chosen freely, but the combination of the three methods is very helpful for determining the tumor's anatomical location and surrounding relationship. The combined imaging examination provides a certain scientific basis for the early detection and accurate treatment of retroperitoneal tumors.

\section{Acknowledgments}

We thank Professor Dean Scott for helping us editing the language.

Funding: None.

\section{Footnote}

Reporting Checklist: The authors have completed the STARD reporting checklist. Available at http://dx.doi.org/10.21037/ tcr-20-3141

Data Sharing Statement: Available at http://dx.doi. org/10.21037/tcr-20-3141

Conflicts of Interest: All authors have completed the ICMJE uniform disclosure form (available at http://dx.doi. org/10.21037/tcr-20-3141). The authors have no conflicts of interest to declare.

Ethical Statement: The authors are accountable for all aspects of the work in ensuring that questions related to the accuracy or integrity of any part of the work are appropriately investigated and resolved. The study was conducted in accordance with the Declaration of Helsinki (as revised in 2013). The study was approved by the Ethics Committee of Peking University International Hospital (approval number 2016-46) and informed consent was taken from all the patients.

Open Access Statement: This is an Open Access article distributed in accordance with the Creative Commons Attribution-NonCommercial-NoDerivs 4.0 International License (CC BY-NC-ND 4.0), which permits the noncommercial replication and distribution of the article with the strict proviso that no changes or edits are made and the original work is properly cited (including links to both the formal publication through the relevant DOI and the license). See: https://creativecommons.org/licenses/by-nc-nd/4.0/.

\section{References}

1. Han Y, Chai Y, Zhang Y, et al. Analysis of surgical treatment for recurrent retroperitoneal sarcoma. China Oncology 2017;27:980-4.

2. Konofaos P, Spartalis E, Moris D, et al. Challenges in the Surgical Treatment of Retroperitoneal Sarcomas. Indian J Surg 2016;78:1-5.

3. Erickson RA, Tretjak Z. Clinical utility of endoscopic ultrasound and endscopic ultrasound-guided fine needle aspiration in retroperitoneal neoplasms. Am J Gastroenterol 2000;95:1188-194.

4. Kaliszewski K, Bednarz W, Łukieńczuk T, et al. Retroperitoneal tumours--analysis of own clinical material-a six-year retrospective study. Hepatogastroenterology 2010;57:47-51.

5. Yu J, Zhu S, Ge Z, et al. Multislice spiral computed tomography in the differential diagnosis of ground-glass opacity. J Cancer Res Ther 2018;14:128-32.

6. Wang SQ, Ren FY, Wang JH, et al. Diagnostic value of multislice spiral computed tomography (CT) combined with CT angiography for intra-abdominal undescended testis secondary seminomas. Cancer Imaging 2019;19:24.

7. Johnstone E, Wyatt JJ, Henry AM, et al. Systematic Review of Synthetic Computed Tomography Generation Methodologies for Use in Magnetic Resonance ImagingOnly Radiation Therapy. Int J Radiat Oncol Biol Phys 2018;100:199-217.

8. Souza D, Lerman I, Halaszynski TM. Ultrasound Technical Aspects: How to Improve Needle Visibility. In: Narouze SN. editor. Atlas of Ultrasound-Guided Procedures in Interventional Pain Management. New York: Springer New York, 2018.

9. Wolf B, Kunert C, Horn LC, et al. Management of Primary Retroperitoneal Mucinous Tumors: A Retrospective Meta-Analysis. Int J Gynecol Cancer 2017;27:1064-71.

10. Füllhase C, Harke N, Niedworok C, et al. Retroperitoneal Tumors in Adults. In: Merseburger A, Burger M. editors. Urologic Oncology. Springer, Cham, 2018. doi: https:// doi.org/10.1007/978-3-319-42603-7_42-1.

11. Liu Q, Changjun WU, Ultrasonography DO. Research Progress of the Application of Ultrasound 
in the Musculoskeletal System. Medical Recapitulate 2017;12:2433-6.

12. Lewis JJ, Leung D, Woodruff JM, et al. Retroperitoneal soft-tissue sarcoma: analysis of 500 patients treated and followed at a single institution. Ann Surg 1998;228:355-65.

13. Schroeder S, Kopp AF, Ohnesorge B, et al. Virtual coronary angioscopy using multislice computed tomography. Heart 2002;87:205-9.

14. Zhao J, Ye Z, Bai B, et al. MSCT diagnosis and differential diagnosis of common pathological subtypes of renal cell carcinoma. Journal of Clinical Radiology 2011;30:210-4.

15. Wu MC, Chin WC, Tsan TC, et al. The benign and Malignant Recognition System of Nasopharynx in MRI

Cite this article as: Feng Y, Zhang W, Luo C. Analysis of the clinical value of multi-slice spiral computed tomography (MSCT), magnetic resonance imaging (MRI) and ultrasound (US) in the diagnosis of retroperitoneal tumors. Transl Cancer Res 2021;10(5):2247-2254. doi: 10.21037/tcr-20-3141 image with Neural-Fuzzy based Adaboost classifier. 2016 2nd International Conference on Information Management (ICIM). 7-8 May 2016; London, UK. IEEE, 2016:47-51.

16. Abe S, Tamakawa M, Andoh M, et al. Lymphoid tumor in the orbit: malignant or benign? MRI, histomorphological and molecular genetic analysis of eight cases. Eur J Plast Surg 2005;27:378-82.

17. Wu RY, Liu K, Wang WH, et al. Patterns of Primary Tumor Invasion and Regional Lymph Node Spread Based on Magnetic Resonance Imaging in Early-Stage Nasal NK/T-cell Lymphoma: Implications for Clinical Target Volume Definition and Prognostic Significance. Int J Radiat Oncol Biol Phys 2017;97:50-9. 